VOL. $69(2004) \quad[297-303]$

\title{
a-T-MENABILITY OF GROUPS ACTING ON TREES
}

\author{
ŚWiatoseaw R. GaL
}

We present some partial results concerning a-T-menability of groups acting on trees.

Various known results are given uniform proofs.

\section{DEFinition of A-T-MENABILITY}

Definition 1.1: Given a metric space $(X, \rho)$, the action $\mathrm{G} \rightarrow \operatorname{Isom}(X)$ is metrically proper if, given $x \in X$, the displacement function $\mathrm{G} \ni g \mapsto \rho(x, g x) \in \mathbb{R}$ is proper. If the group action is set we say that $X$ is metrically proper.

The above property does not depend on a choice of a point $x$.

Definition 1.2: (M. Gromov) A locally compact, second countable, compactly generated group $\mathrm{G}$ is $a$ - $T$-menable if there exists metrically proper isometric G-action on some affine Hilbert space.

REMARK. a-T-menability is equivalent to the existence of $C_{0}$-approximate unity consisting of positive definite functions. The latter is called in the literature the Approximation Property of Haagerup.

Throughout this paper by representation we mean isometric affine action. By a subgroup we always mean a closed subgroup.

\section{Motivation}

We are motivated by the following Theorems:

THEOREM 2.1. ([2, Theorem 6.2.8]) Let $\Gamma$ be a countable group acting on a tree without inversions, with finite edge stabilisers. If vertex stabilisers in $\Gamma$ are a- $T$-menable, then so is $\Gamma$.

THEDREM 2.2. ([2, Example 6.1.6]) If $\mathrm{N}$ is a-T-menable and $\mathrm{G} / \mathrm{N}$ is amenable then $\mathrm{G}$ is a-T-menable.

On the other hand:

Received 30th September, 2003

Partially supported by a KBN grant 2 P03A 01725 . The author would like to thank Tadeusz Januszkiewicz for his helpful information, Agnieszka for her hospitality in Vienna and finally Jan Dymara for carefully reading preliminary version of this paper and his assistance in improving the presentation.

Copyright Clearance Centre, Inc. Serial-fee code: 0004-9727/04 \$A2.00+0.00. 
TheOREM 2.3. ([5, Chapter 8 Lemma 6]) Every isometric affine representation of $\mathrm{G}=S L_{2} \mathbb{Z} \times \mathbb{Z}^{2}$ has a $\mathbb{Z}^{2}$-fixed point.

The group $G$ acts on a tree as it can be decomposed as $\left(\mathbb{Z} / 4 \propto \mathbb{Z}^{2}\right) *_{\mathbf{Z} / 2 \times \mathbb{Z}^{2}}\left(\mathbb{Z} / 6 \times \mathbb{Z}^{2}\right)$. The factors are a-T-menable by Theorem 2.2 , since $\mathbb{Z}^{2}$ is a-T-menable as it acts on $\mathbb{R}^{2}$. On the other hand $S l_{2} \mathbb{Z}$ is a-T-menable by the result of Haagerup [4]. Therefore extra assumptions about finitness of edge stabilisers (in Theorem 2.1) or amenability of the quotients (in Theorem 2.2) cannot be simply weakened to a-T-menability.

We believe that a-T-menability is a property of representations (see next section for definitions), rather than that of groups. Therefore in this paper we shall concentrate on a question: what are the conditions under which given proper affine representations of two groups extend to one of their product with amalgamation, rather than whether there exist some such representation that extend.

The most naive observation is that if there is a proper representation of a free product with amalgamation, the restrictions are.proper representations of the factors that coincide on the common subgroup. It is not known whether the reverse holds, however we shall prove some results in this direction.

The main result of this paper is Theorem 5.4. As a result we strengthen a result from [2] (see Section 6). Constructions, we give, are purely geometric. We shall also give an affirmative answer to the question of A. Valette, whether Baumslag-Solitar groups are a-T-menable. This was originally done (using another approach) in [3].

\section{ACTIONS ON TREES IN GENERAL}

Before we examine the case of a free product with amalgamation, let us restate a general observation of Haagerup [4] and its easy generalisations.

Let $T$ be a tree. By $E$ we denote a vector space of functions on edges of $\mathrm{T}$ with finite support. By $V$ we denote the affine space of functions on vertices of $T$ with finite support and total mass one. The structure of affine space is given as follows: $\delta_{v}-\delta_{w}$ is equal to the characteristic function of the segment joining vertices $v$ and $w$ (with the appropriate signs with respect so some auxiliary orientation on the edges of $T$ ).

DEFINITION 3.1: Let $U(T)$ be an affine Hilbert space completion of $V$ defined above.

There is an obvious $\operatorname{Aut}(T)$ action of the group of cellular automorphisms of $T$ on $U(T)$, with an $\operatorname{Aut}(T)$-equivariant isometric embedding of $T$. An immediate consequence of the construction is

PROPOSITION 3.2: If $\Gamma$ acts metrically properly on a tree $T$ then $\Gamma$ is a-T-menable.

In particular $\mathrm{Sl}_{2} \mathbb{Z}=\mathbb{Z} / 4 *_{\mathbf{z} / 2} \mathbb{Z} / 6$ is a-T-menable.

If $T$ is locally finite, the (topological) group of all cellular automorphisms of $T$ is a-T-menable. Even if $\Gamma$ acts effectively on $T$, the inclusion $\Gamma \rightarrow \operatorname{Aut}(T)$ in general is not closed (therefore we cannot conclude that $\Gamma$ is a-T-menable). However we have 
Proposition 3.3. If $\Gamma$ acts on a locally finite tree $T$ and there exists an affine representation of $\Gamma$ on an affine Hilbert space $W$, such that stabiliser of any vertex acts properly, then $U(T) \oplus W$ is $\Gamma$-proper.

Proof: Given $g_{n} \in \Gamma$ with bounded displacement (as acting on $T$ ), for any vertex $v$ the distance from $g_{n} v$ to $v$ is bounded. Since there are only finitely many such vertices, one can take a subsequence such that $g_{n} v$ is constant. Stabilisers of vertices act properly on $W$, thus $\left\{g_{n}\right\}$ is relatively compact.

Proposition 3.4. If $\Gamma$ acts on a locally finite tree $T$ and for each vertex $v$ some affine proper representation of $\mathrm{Stab}_{v}$ on $W_{v}$ extends to a (perhaps non-proper) affine representation of $\Gamma$, then $\Gamma$ is a-T-menable.

Proof: This is obvious if the quotient $\Gamma \backslash T$ has finitely many vertices, since then the sum of the representations corresponding to any lifts will fulfill the assumptions of Proposition 3.3.

Since there is no infinite sum operation in the category of affine Hilbert spaces, the construction will depend on choices made.

Let $\left\{v_{k}\right\}$ be a sequence of representatives of the vertices of the quotients. Let $K_{k}$ be an exhausting sequence of compact subsets of $\Gamma$, let $x_{k} \in W_{v_{k}}$,

$$
a_{k}=k^{2}+\sup \left\{\left\|x_{k}-g x_{k}\right\|^{2}: g \in K_{k}\right\} .
$$

Define a norm on $\prod_{k} W_{v_{k}}$ in a following way:

$$
\|y-z\|^{2}:=: \sum a_{k}^{-2}\left\|y_{k}-z_{k}\right\|^{2} .
$$

Let $W$ be the completition of the afiine space

$$
\left\{y \in \prod_{k} W_{v_{k}}:\|y-x\|<\infty\right\} .
$$

The choices are made in a such way that $\Gamma$ acts diagonally on $W$ and there are $\Gamma$-equivariant projections (up to scalar change of norm) to each of $W_{v_{k}}$.

Note. The proof of Proposition 3.4 follows the standard proof of the fact that direct limit of a-T-menable groups is also a-T-menable.

The example of $S l_{2} \mathbb{Z} \propto \mathbb{Z}^{2}$ shows that there are some obstructions for a representation to extend from the subgroup. In the terms of group cohomology, inclusion of groups need not induce epimorphisms on the level of the first cohomology.

\section{AfFine RePresentations AND SUbGroups}

If $V$ is G-invariant subspace of $W$ then $W / V$ is a linear G-representation (the coset $V$ is a fixed point), therefore $V$ is metrically proper if and only if $W$ is. In general, there 
is no minimal invariant subspace. Let $\mathrm{H}<\mathrm{G}$. Assume $V \subset W$ is $\mathrm{H}$-invariant subspace. Fix $x \in W$. Define

$$
\psi_{(V \subset W)}: H \backslash G \ni H g \mapsto\|g x+V\| \in \mathbb{R}
$$

(where $g x+V$ is a coset of $g x$ in $W / V)$. If $\psi_{(V \subset W)}$ is proper we say that $(V \subset W)$ is $H \backslash G$-proper. The definition does not depend on the choice of $x$. If $H=\{e\}<G$, and $V$ is any point, then $(V \subset W)$ is $H \backslash G$-proper exactly if $W$ is G-proper.

LEMmA 4.1. Let $\mathrm{G}_{1}<\mathrm{G}_{2}<\mathrm{G}_{3}$. If there are $W_{1} \subset W_{2} \subset W_{3}$, such that $W_{i}$ is $G_{i}$ invariant and $\left(W_{i} \subset W_{i+1}\right)$ is $G_{i} \backslash G_{i+1}$-proper then $\left(W_{1} \subset W_{3}\right)$ is $G_{1} \backslash G_{3}$-proper.

Proof: If $\psi_{\left(W_{1} \subset W_{3}\right)}\left(G_{1} g_{n}\right)$ is bounded, then $\psi_{\left(W_{2} \subset W_{3}\right)}\left(G_{2} g_{n}\right)$ is bounded. Therefore $g_{n}=g_{n}^{\prime} h_{n}$ where $g_{n}^{\prime} \in \mathrm{G}_{2}$ and $\left\{h_{n}\right\}$ is relatively compact. By the triangle inequality

$$
\left\|g_{n}^{\prime} x-x\right\| \leqslant\left\|g_{n}\left(h_{n}^{-1} x-x\right) \mid+\right\| g_{n} x-x\|+\| h_{n}^{-1} x-x \|,
$$

so $\psi_{\left(W_{1} \subset W_{2}\right)}\left(\mathrm{G}_{1} g_{n}^{\prime}\right)$ is bounded, therefore $g_{n}^{\prime}=g_{n}^{\prime \prime} h_{n}^{\prime}$, where $g_{n}^{\prime \prime} \in \mathrm{G}_{1}$ and $\left\{h_{n}^{\prime}\right\}$ is relatively compact. Finally $g_{n}=g_{n}^{\prime \prime}\left(h_{n}^{\prime} h_{n}\right)$.

Unfortunately it is not known whether for any $\mathrm{H}<\mathrm{G}$ and proper G-representation $W$ there exist $\mathrm{H}$-invariant subspace $V$, such that $(V \subset W)$ is $H \backslash G$-proper. The cases when it does happend are discussed in the following sections.

\section{Free products with amalgamation}

If $\Gamma=G_{1} *_{H} G_{2}$ then, according to Serre theory [6], a graph $T$ with the set of vertices equal to $\Gamma / G_{1} \cup \Gamma / G_{2}$ and the set of edges equal to $\Gamma / H$, with inclusion as incidence relation, is a tree (on which $\Gamma$ acts on the left). The representations of $G_{1}$ and $G_{2}$ on $U(T)$ have global fixed points.

Let $\mathrm{H}$ be a common subgroup in $\mathrm{G}_{1}$ and $\mathrm{G}_{2}$. Let $W_{i}$ be $\mathrm{G}_{i}$-representations. Let $W$ be their common $H$-invariant subspace. Define $\mathcal{H}_{i}:=W_{i} / W$. Inductively decompose $\uparrow_{H}^{G_{i}} \mathcal{H}_{\omega}$ (where $\omega$ is a sequence of $1 \mathrm{~s}$ and 2s) with respect to $H$ as $\mathcal{H}_{\omega} \oplus \mathcal{H}_{i \omega} . G_{1}$ acts on

$$
\mathcal{H}_{2}^{\bullet}=\left(\mathcal{H}_{2} \oplus \mathcal{H}_{12}\right) \oplus\left(\mathcal{H}_{212} \oplus \mathcal{H}_{1212}\right) \oplus \ldots
$$

and

$$
\mathcal{H}_{1}^{\circ}=\left(\mathcal{H}_{21} \oplus \mathcal{H}_{121}\right) \oplus \ldots,
$$

$\mathrm{G}_{2}$ acts on $\mathcal{H}_{1}^{\circ}=\left(\mathcal{H}_{1} \oplus \mathcal{H}_{21}\right) \oplus \cdots$ and $\mathcal{H}_{2}^{\circ}=\left(\mathcal{H}_{12} \oplus \mathcal{H}_{212}\right) \oplus \cdots$. Both representations of $\mathrm{H}$ on $\mathcal{H}_{i}^{\bullet}$ coincide.

Definition 5.1. Let

$$
W_{\Gamma}=W \oplus \mathcal{H}_{1}^{\bullet} \oplus \mathcal{H}_{2}^{\bullet}=W_{1} \oplus \mathcal{H}_{1}^{\circ} \oplus \mathcal{H}_{2}^{\bullet}=W_{2} \oplus \mathcal{H}_{1}^{\bullet} \oplus \mathcal{H}_{2}^{\circ}
$$

An immediate consequence from the construction is 
THEOREM 5.2. Let $\mathrm{H}<\mathrm{G}_{i}, i=1,2$. Let $W_{i}$ be $\mathrm{G}_{i}$-representations. Let $W$ be their common $\mathrm{H}$-invariant subspace. Let $\Gamma=\mathrm{G}_{1} *_{\mathrm{H}} \mathrm{G}_{2}$. Then $W_{i}$ and $W$ are respectively $\mathrm{G}_{i}-$ and $\mathrm{H}$-invariant subspaces of $W_{\Gamma}$.

NotE. Although there is no way to induce an affine representation, $W_{\Gamma}$ is morally equal to $\uparrow_{G_{1}}^{\Gamma} W_{1} \oplus \uparrow_{G_{2}}^{\Gamma} W_{2} / \uparrow_{H}^{\Gamma} W$. If $W^{\prime} \subset W$ is another $\mathrm{H}$-invariant subspace, then $W_{\Gamma}$ is not a $\Gamma$-invariant subspace of $W_{\Gamma}^{\prime}$ (constructed from the triple $W_{1} \supset W^{\prime} \subset W_{2}$ ).

A straightforward consequence of Proposition 3.3 and Theorem 5.2 is the following.

Corollary 5.3. If $\mathrm{H}$ is of finite index in $\mathrm{G}_{1}$ and $\mathrm{G}_{2}$ and if there are metrically proper representations of $G_{i}$ that coincide when restricted to $H$, then $G_{1} *_{H} G_{2}$ is a-Tmenable.

EXAmple. $\left([2, \mathbf{1}]\right.$.) The torus group $\Gamma_{p, q}=\left\langle x, y \mid x^{p}=y^{q}\right\rangle$ is a-T-menable.

THEOREM 5.4. With the assumptions of Theorem 5.2, if $W_{i}$ is proper affine $G_{i}$ representation and $\left(W \subset W_{i}\right)$ is $H \backslash G_{i}$-proper for $i=1,2$ then

(1) $W_{\Gamma} \oplus U(T)$ is $\Gamma$-proper,

(2) if $\mathrm{H}^{\prime}<\mathrm{G}_{1}$ and $V$ is proper $\mathrm{H}^{\prime}$-space such that $\left(V \subset W_{1}\right)$ is $\mathrm{H}^{\prime} \backslash \mathrm{G}_{1}$-proper, then $\left(V \subset W_{\Gamma} \oplus U(T)\right)$ is $\mathrm{H}^{\prime} \backslash \Gamma$-proper.

PROOF: In fact (1) is a special case of (2). Therefore we shall prove (2).

Given $\gamma_{n} \in \Gamma$ such that $\psi_{(W, V)}\left(\gamma_{n}\right)$ is bounded. Define the length function $\ell: \Gamma \rightarrow \mathbb{N}$ by $\ell_{\mid H} \equiv 0, \ell(\gamma)=\min \left\{\ell(\eta)+1 \mid \gamma=\eta g\right.$, where $\left.g \in \mathrm{G}_{1} \cup \mathrm{G}_{2}\right\}$. This function is equal to the distortion of the action on $U(T)$. Therefore we may find a subsequence such that $\ell\left(\gamma_{n}\right)=k$.

If $k \leqslant 1$ there is nothing to prove. If all but finitely many $\gamma_{n} \in \mathrm{G}_{2}$ we have to use Lemma 4.1 .

Let $x \in V$. Define $\varphi_{x}(\gamma)$ to be the component of $\gamma x$ in $\underset{|\omega|=l(\gamma)}{\bigoplus_{\omega}} \mathcal{H}_{\omega}$.

LEMMA 5.5. If $\gamma=\gamma_{1} \gamma_{2}, \ell(\gamma)=\ell\left(\gamma_{1}\right)+\ell\left(\gamma_{2}\right)$ and $\ell\left(\gamma_{2}\right) \geqslant 1$, then $\left\|\varphi_{x}(\gamma)\right\|$
$=\left|\varphi_{x}\left(\gamma_{2}\right)\right|$.

PROOF: Without loss of generality $\ell\left(\gamma_{1}\right)=1$. From the definition of induced representation $\gamma_{1} \mathcal{H}_{\omega} \perp \mathcal{H}_{\omega}$, therefore $\varphi_{x}(\gamma)=\gamma_{1} \varphi_{x}\left(\gamma_{2}\right)$.

Now we proceed by induction on $k$ as follows. We define $\eta_{n}$ such that $\gamma_{n}=\eta_{n} g_{n}$ $\left(g_{n} \in \mathrm{G}_{i}\right)$ and $l\left(\eta_{n}\right)=k-1$.

From Lemma 5.5 we see $\left\|\gamma_{n} x-x\right\| \geqslant\left\|\varphi_{x}\left(\gamma_{n}\right)\right\|=\left\|\varphi_{x}\left(g_{n}\right)\right\|=\psi_{\left(w_{i}, w\right)}\left(g_{n}\right)$. Therefore $\left\{g_{n}\right\}$ is relatively compact. Since $\eta_{n} x=\gamma_{n} x-\eta_{n}\left(g_{n} x-x\right)$ and, by induction assumption, $\psi_{\left(V, W_{\Gamma}\right)}$ is proper when restricted to cosets of elements of length smaller than $k$, we obtain the claim. 


\section{GROUPS THAT ACT ON TREES WITH FINITE EDGE STABILISERS}

The first case, where it is easy to fulfill the assumptions of the Theorem 5.4 is when $\mathrm{H}$ is finite, since then one can find a fixed point of any $H$-representation simply taking the centre of mass $*$ of any orbit. The pair $\left(\{*\} \subset W_{i}\right)$ is $H \backslash G_{i}$ proper if and only if $W_{i}$ is proper $G_{i}$-space. Summarising this:

Proposition. ([2, Proposition 6.2.3 (1)]) Let $\mathrm{G}_{1}, \mathrm{G}_{2}$ be two grups containing finite subgroup $H$, and let $\Gamma=\mathrm{G}_{1} *_{H} \mathrm{G}_{2}$ be the corresponging amalgamated product. If $\mathrm{G}_{1}$ and $\mathrm{G}_{2}$ are a- $T$-menable, then so is $\Gamma$.

Theorem 2.1 is an easy consequence of the above [2].

\section{BAUMSLAG-SolitAR GROUPS ANT THEIR CERTAIN GENERALISATIONS}

The second easy case occurs when $H$ is of finite index in $G_{i}{ }^{\prime} s$. Then any pair is automatically $H \backslash G_{i}$ proper.

Let us recall some definitions from [3]. Let $G \subset \mathfrak{N}$ be a closed subgroup of a locally compact, compactly generated, topological group $\mathfrak{N}$. Let $i_{k}: \mathrm{H} \rightarrow \mathrm{G}(k=1,2)$ be two inclusions onto finite index open subgroups, which are conjugated by an automorphism $\phi$ of $\mathfrak{N}$.

Definition 7.1: The $\mathfrak{N}$-BS group $\Gamma$ is the group derived from $\left(G, H, i_{1}, i_{2}\right)$ by the (topological) HNN construction.

ThEOREM 7.2. ([3].) If $\mathfrak{N}$ is a-T-menable then $\mathfrak{N}$-BS groups are a- $T$-menable.

Proof: We mimic the proof $[2,6.2 .7]$ for the case of a HNN extension, where the edge stabiliser is finite.

STEP 1. Let $\Gamma_{0}$ be a fundamental group of the following tree:

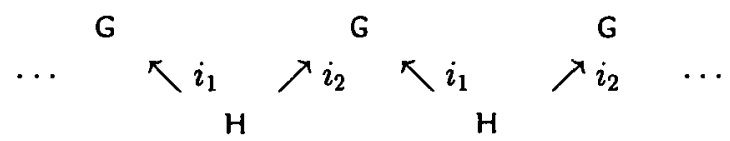

We have to find consistent representations of different copies of $G$. The Hilbert space in each case will be the one on which $\mathfrak{N}$ acts properly. The $k$-th copy acts by $\phi^{k}(\cdot)$, where $\phi$ is the automorphism of $\mathfrak{N}$ that conjugates $i_{1}$ and $i_{2}$.

By induction, each of

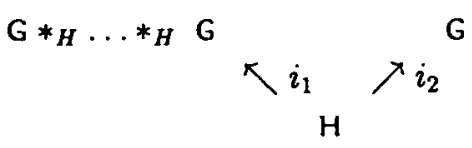

satisfies assumptions of Theorem 5.4 (alternatively: by Theorem 5.2 and induction we construct representation of $\Gamma_{0}$ and then use Proposition 3.4). It is easy to show [2, 
Proposition 6.1.1] that an increasing union of open a-T-menable subgroups is again a-Tmenable. Therefore $\Gamma_{0}$ is a-T-menable.

STEP 2. $\Gamma=\Gamma_{0} \times \mathbb{Z}$, where $\mathbb{Z}$ acts through the shift. Therefore $\Gamma$ is an extension of an a-T-menable group with amenable quotient, so $\Gamma$ is a-T-menable by Theorem 2.2 .

\section{REFERENCES}

[1] C. Béguin and T. Ceccherini-Silberstein, 'Formes faibles de moyenabilité pour les gruupes à un relateur', Bull. Belg. Math. Soc. Simon Stevin 1 (2000), 135-148.

[2] P-A. Cherix, M. Cowling, P. Jolissant, P. Julg and A. Valette, Groups with the Haagerup property (Gromov's a-T-menability) (Birkhäuser Verlag, Basel, 2001).

[3] S.R. Gal and T. Januszkiewicz, 'New a-T-menable HNN-extensions', J. Lie Theory 13 (2003), 383-385.

[4] U. Haagerup, 'An example of non-nuclear $C^{*}$-algebra which has the metric approximation property', Invent. Math. 50 (1979), 279-293.

[5] P. de la Harpe and A. Valette, 'La properiété (T) de Kazhdan pour les groupes localement compacts', Astérisque 157 (1989), 158.

[6] J.P. Serre, Trees (Springer-Verlag, Berlin, New York, 1980).

Department of Mathematics

Wroclaw University

pl. Grunwaldzki $2 / 4$

50-384 Wroclaw

Poland

http://www.math.uni.wroc.pl sgal/ 